\title{
A catalogue of low-mass X-ray binaries in the Galaxy, LMC, and SMC (Fourth edition)^
}

\author{
Q. Z. Liu ${ }^{1,2}$, J. van Paradijs ${ }^{2}$, and E. P. J. van den Heuvel ${ }^{2}$ \\ ${ }^{1}$ Purple Mountain Observatory, Chinese Academy of Sciences, Nanjing 210008, PR China \\ e-mail: qzliu@pmo.ac.cn \\ 2 Astronomical Institute Anton Pannekoek, University of Amsterdam, Kruislaan 403, 1098 SJ Amsterdam, The Netherlands
}

Received 15 February 2007 / Accepted 29 March 2007

\begin{abstract}
We present a new edition of the catalogue of the low-mass X-ray binaries in the Galaxy and the Magellanic Clouds. The catalogue contains source name(s), coordinates, finding chart, X-ray luminosity, system parameters, and stellar parameters of the components and other characteristic properties of 187 low-mass X-ray binaries, together with a comprehensive selection of the relevant literature. The aim of this catalogue is to provide the reader with some basic information on the X-ray sources and their counterparts in other wavelength ranges ( $\gamma$-rays, UV, optical, IR, and radio). Some sources, however, are only tentatively identified as low-mass X-ray binaries on the basis of their X-ray properties similar to the known low-mass X-ray binaries. Further identification in other wavelength bands is needed to finally determine the nature of these sources. In cases where there is some doubt about the low-mass nature of the $\mathrm{X}$-ray binary this is mentioned. Literature published before 1 October 2006 has, as far as possible, been taken into account.
\end{abstract}

Key words. binaries: close - stars: late-type - X-rays: binaries - catalogs

\section{Introduction}

An X-ray binary contains either a neutron star (NS) or a black hole $(\mathrm{BH})$ accreting material from a companion star. Thus, white dwarf (WD) systems are not included among the X-ray binaries. $\mathrm{X}$-ray binaries can be further divided into two different classes, regardless the nature of the compact object, according to the mass of the companion star: high-mass X-ray binaries (HMXB) and low-mass X-ray binaries (LMXB). In a previous paper we presented an up-to-date catalogue of high-mass X-ray binaries (Liu et al. 2006).

The secondary of LMXB systems is a low-mass (in general $M \leq 1 M_{\odot}$ ) star, which transfers matter by Roche-lobe overflow. Among the low-mass companion stars we find white dwarfs, late-type main-sequence stars, A-type stars and F-G-type subgiants. The last-mentioned category of companion stars may well be the mass-transfer remnants of stars that originally were of intermediate mass $\left(M \sim 1.5\right.$ to $\left.4 M_{\odot}\right)$ as has been suggested for Cygnus X-2 (cf. Podsiadlowski \& Rappaport 2000; Tauris et al. 2000). The optical counterparts of LMXBs are intrinsically faint objects. The spectra of most of them show a few characteristic emission lines superposed on a rather flat continuum. The optical continuum of LMXBs is dominated by the emission from an accretion disk around the compact star, which is predominantly the result of reprocessing of a fraction of the X-rays into optical photons in the disk. The contribution from the secondary is generally negligible. On occasions, however, the presence of the secondary can be discerned in the spectrum (or colors) of the LMXBs. This particularly is the case for systems with donors

* Table 1 is only available in electronic form at the CDS via anonymous ftp to cdsarc.u-strasbg.fr $(130.79 .128 .5)$ or via http://cdsweb.u-strasbg.fr/cgi-bin/qcat?]/A+A/469/807 that are or started out as intermediate mass stars, such as Her X-1 and Cyg X-2. For a full understanding of LMXBs in various aspects one can refer to the book by Lewin \& van der Klis (2006).

The classification as LMXB is mainly based on the spectra obtained from an optical identification, and/or on the mass function from X-ray pulse arrival time measurements. If neither is available, a classification may be inferred based on the similarity of the X-ray properties to other identified systems. An unidentified system is classified as a LMXB containing a neutron star if one or more of the following properties are observed:

- type I X-ray bursts (which to date have only been seen from neutron stars in LMXBs);

- a 1-10 keV soft spectrum with a characteristic temperature of $5-10 \mathrm{keV}$ and/or

- an orbital period is less than about $12 \mathrm{~h}$.

Six years after the publication of the previous (3rd) edition (Liu et al. 2001), the amount of new literature and the number of new objects to be included have again grown so much that it seems worthwhile to publish an updated catalogue. This new catalogue contains 187 sources, 44 new low-mass X-ray binaries in addition to the 143 sources listed in the previous catalogue. We briefly recall some of the developments that, over the past six years, have had (and still have) a major impact on this catalogue.

Due to the much increased sensitivity and spatial resolution achievable with the Chandra and the XMM-Newton $\mathrm{X}$-ray observatories, as well as with the unparalleled resolution of the Hubble Space Telescope and large ground-based radio telescopes, more accurate positions of X-ray binaries have been determined, resulting in the unambiguous discovery of the optical and/or IR counterpart to some X-ray sources. Moreover, the number of LMXBs in external galaxies is also rapidly 
increasing, e.g., the X-ray binaries in NGC 720 (Jeltema et al. 2003) and NGC 1399 (Angelini et al. 2001). Barnard et al. (2003) even discovered a Z source low-mass X-ray binary, RX J0042.6+4115, in M31. Most of the ultra-luminous X-ray sources in elliptical galaxies (Liu \& Mirabel 2005) are believed to be LMXBs with a black hole. It has been proposed that the collective X-ray luminosity of low-mass X-ray binaries can be used as a stellar mass indicator for the host galaxy (Gilfanov 2004).

Prior to Chandra and $X M M$, there were 12 bright cluster LMXBs (now 13, all thought to have neutron stars as primaries) and 57 faint X-ray sources known in the Galactic globular cluster systems (Verbunt 2001). A few of the latter had been identified with cataclysmic variables, and some were thought to be quiescent LMXBs (qLMXBs) containing neutron stars (Verbunt et al. 1984). Much more quiescent low-mass X-ray binaries have been identified in several globular clusters using Chandra or XMM X-ray observations (e.g. NGC 6440, Heinke et al. 2003). These qLMXBs, however, have not been included in this catalogue.

Since its launch in 2002, INTEGRAL has been revealing hard $\mathrm{X}$-ray sources that were not easily detected in earlier soft X-ray (typically $\leq 10 \mathrm{keV}$ ) observations (Winkler et al. 2003). Hard $\mathrm{X}$-rays are not easily absorbed by matter and thus are highly penetrating. Such radiation is, therefore, ideal for probing highenergy emitting sources in dense regions. INTEGRAL has been performing a regular survey of the Galactic plane and a deep exposure of the Galactic Center as part of its Core Program. Bird et al. (2007) have recently published an updated catalogue of the INTEGRAL X-ray sources. The catalogue contains 421 point $X$-ray sources, some of which are included in our catalogue. For the details on new INTEGRAL sources please refer to the web page of Jerome Rodriguez (http://isdc . unige.ch/ rodrigue/html/igrsources.html).

The aim of our catalogue is to provide some basic information on the X-ray sources and their counterparts, as well as the binary properties of the system in question, and easy access to the recent literature. No attempt has been made to compile complete reference lists. Much effort has been made to avoid errors and to keep the information up to date. Nevertheless, the authors are well aware that this edition too may contain errors and may be incomplete.

\section{Description of the table}

Table 1 lists the 187 LMXBs. The format of Table 1 is similar to the previous edition (Liu et al. 2001), of which the present catalogue is meant to be an update. In the table the sources are ordered according to the right ascension in the source names; part of the (mainly numerical) information on a source is arranged in six columns, below which additional information is provided for each source in the form of key words with reference numbers [in square brackets]. When a result is unreliable, a colon (:) or a question mark (?) will follow the adopted entry. The columns have been arranged as follows.

In Col. 1 the first line contains the source name, which contains an indication on its sky location, according to the conventional source nomenclature of space experiments in which the source was detected, hhmm \pm ddd or hhmm.m \pm ddmm. Here hh, $\mathrm{mm}$, and ss indicate the hours, minutes and seconds of right ascension, ddd the declination in units of 0.1 degree (in a small number of cases, the coordinates shown in the name are given with more, or fewer, digits). The prefix $\mathrm{J}$ indicates a name based on J2000 coordinates. Otherwise, 1950 coordinates were used in the name. An alternative source name is given in the second line.
In the third line of Col. 1, the source types are indicated with a letter code, as follows:

- A: known atoll source (25);

The majority of systems in the catalogue are unclassified. However, advances in our knowledge and understanding of the properties of the neutron-star X-ray binaries indicate that the majority of the unclassified systems are likely to be Atolllike, although a small subclass of lower-luminosity sources is possible (Fender \& Hendry 2000).

- B: X-ray burst source (84);

There are a dozen of superbursts detected from ten sources: one from $4 \mathrm{U}$ 1735-44, Ser X-1, KS 1731-260, 4U 1820303, GX 3+1, 4U 0614+091, 4U 1608-522, 4U 1254, SLX $1735-269$ and three from $4 \mathrm{U} 1636-536$. It is thought that unstable carbon burning (Woosley \& Taam 1976; Strohmayer \& Brown 2002) in a heavy-element ocean (Cumming \& Bildsten 2001), possibly combined with photodisintegration-triggered nuclear energy release (Schatz et al. 2003), is responsible for most superbursts.

- D: "dipping" low-mass X-ray binary (22);

- E: eclipsing or partially eclipsing low-mass X-ray binary (13);

- G: globular-cluster X-ray source (14);

- M: microquasar (14);

- P: X-ray pulsar (12); These sources include the 7 currently known accretion-driven millisecond X-ray pulsars (IGR J00291+5934, XTE J0929-314, XTE J1751-305, XTE J1807-294, SAX J1808.4-3658, XTE J1814-338, and HETE J1900.1-2455).

- R: radio loud X-ray binary (56);

- T: transient X-ray source (103);

- U: ultra-soft X-ray spectrum (18); These sources include black-hole candidates; some "extreme ultra-soft" (EUS) sources may be white dwarfs on whose surface steady nuclear burning takes place.

- Z: Z-type source (8), including the possible new Z source, XTE J1701-462 (Homan et al. 2006).

Column 2 contains the right ascension (RA) and declination (Dec) of the source for equinox J2000.0 in the first two lines. RA is given as hhmmss.s to an accuracy of $0.1 \mathrm{~s}$, Dec is given in ${ }^{\circ \prime \prime}$, to an accuracy of 1 " (in a small number of cases, the coordinates are given with more, or fewer, digits). The third line gives the galactic longitude and latitude to an accuracy of $0.1^{\circ}$ (except for sources close to the galactic center, where these coordinates are given to $0.01^{\circ}$ ). A reference for the source position is specified in the table after "Pos.". In the parentheses following the "pos.", we provide some information on the type of observation from which the source position has been derived. The following abbreviations are used: o, optical; $\mathrm{x}, \mathrm{X}$-ray; r, radio; IR, infrared. Following the type of observation, we give an indication of the accuracy of this position, in the form of equivalent (90 percent confidence level) error radii, but in several cases this can only be considered an approximation (e.g. when the error box is not circular).

The first and second lines of Col. 3 give the names of the optical counterpart to an X-ray source. The third line contains a reference, in which the finding chart to the X-ray source can be found. An asterisk followed by a number or letter refers to the star number used in the finding chart; "star" refers to a star in the finding chart that has not been assigned a number or letter. If there is only a reference but nothing else specified in the first two lines, that means there is no optical counterpart to be found. Many optical counterparts have been indicated with a 
variable-star name, as given in the General Catalogue of Variable Stars and in recent name lists of variable stars as published regularly in the IAU Information Bulletin on Variable Stars, or a number in a well-known catalogue (e.g. HD, SAO). For X-ray sources in globular clusters, the cluster name is given, in addition to the name of a stellar optical counterpart.

The fourth column contains some photometric information on the optical counterpart. In the first line, the apparent visual magnitude, $V$, and the color indices $B-V$, and $U-B$, are listed. The second line contains the estimate of the interstellar reddening, $E_{B-V}$. Some of them are derived from the best-fit column density $N_{\mathrm{H}}\left(\right.$ in $\mathrm{cm}^{-2}$ ) of their X-ray spectrum, through the following relation (Predehl \& Schmitt 1995),

$$
\begin{aligned}
A_{V} & =\frac{N_{\mathrm{H}}}{1.79 \times 10^{21}} \\
E(B-V) & =A_{V} / 3.2 .
\end{aligned}
$$

In Col. 5, the maximum X-ray flux, or the range of observed $\mathrm{X}$-ray fluxes $(2-10 \mathrm{keV}$, unless otherwise indicated), is given, in units of

$$
\begin{aligned}
1 \mu \mathrm{Jy} & =10^{-29} \mathrm{erg} \mathrm{cm}^{-2} \mathrm{~s}^{-1} \mathrm{~Hz}^{-1} \\
& =2.4 \times 10^{-12} \mathrm{erg} \mathrm{cm}^{-2} \mathrm{~s}^{-1} \mathrm{keV}^{-1}
\end{aligned}
$$

The first line in Col. 6 gives the orbital period in hours (in a small number of cases with very wide orbit, the unit of orbital period is in days, indicated with a " $d$ " after the number). The second line contains the pulse period for X-ray pulsars, in seconds. The third line contains a reference in which the orbital and/or pulse periods were detected.

\section{Conclusions and remarks}

The current version of this catalogue provides tabulated data and references for 187 objects, including 44 newly discovered LMXBs ( 2 previously listed in our high-mass X-ray binary catalogue), as well as 143 "old" ones listed in the previous catalogue. Compared with the 3rd edition, the number of LMXBs in the Galaxy listed has increased by $\sim 30 \%$. Among the 187 LMXB candidates, we find 3 symbiotic LMXBs with M-type giant companion (4U 1700+24, 4U 1954+319, and GX 1+4), 15 known and suspected ultra-compact X-ray binaries (UCXBs) (4U 0614+091, 4U 0919-54, 1A 1246-588, 4U 1543624, 4U 1626-67, XTE J1709-267, EXO 1745-248, 4U 181212, 4U 1820-30, 4U 1823-00, XB 1832-330, 4U 1850-087, $4 \mathrm{U}$ 1905+000, 4U 1916-05, and CXO J212958.1+121002), and 14 sources with millisecond burst oscillations. UCXBs are those systems with orbital periods $<80 \mathrm{~min}$, which is the minimum period for LMXBs with hydrogen-rich main sequence donors. In these ultracompact binaries the mass donor must be a nondegenerate hydrogen-deficient star or a white dwarf (e.g. Verbunt $\&$ van den Heuvel 1995). In addition to the 7 millisecond X-ray pulsars, there are $14 \mathrm{X}$-ray sources with millisecond burst oscillations. It is certain that the sources are neutron stars with millisecond spin periods, since Chakrabarty et al. (2003) found compelling evidence that burst oscillation is the same as the $\mathrm{X}$-ray pulsation.

We wish to emphasize here that some sources listed in this catalogue are still uncertain. They need to be regarded with caution in view of all the further work needed. Some sources are tentatively classified as low-mass X-ray binaries due to the similarity of the X-ray properties to other identified systems. No counterpart at other bands has been found.
We would like to make some remarks on several individual sources. Both 2S 1803-245 and XTE J1806-246 have been listed in the 3rd edition. Marshall et al. (1998), reported that the bright, soft X-ray transient, XTE J1806-246, is at a position consistent with that of $2 S$ 1803-245. Therefore, in the new version of the catalogue, they are regarded as the same source. Similarly, the BeppoSAX source, SAX J1748.9-2021, is the only one in the Uhuru error boxes of 4U 1745-203 (in't Zand et al. 1999), also regarded as the identical source.

The following six X-ray sources are no longer listed in this LMXB catalogue. Three previous LMXBs, MXB 0656-072, SAX J1819.3-2525/V4641 Sgr, and GRS 1736-297, have been listed in the HMXB catalogue, because of their HMXB properties (Liu et al. 2006). In SIMBAD databases, SAX J0835.9+5118 is an X-ray flash source, GRB 990520, instead of an X-ray burster, thus excluded from the LMXB catalogue. For the other two previously proposed low-mass X-ray binaries, GRS 1734-292 and GT 2318+620, new optical and radio observations provide substantial evidences to rule out their galactic origin and point towards identification as active extragalactic sources (Marti et al. 1998; Paredes et al. 2004).

The following two X-ray sources, 4U 1954+319 and 1A 1246-588, were previously listed as HMXBs but are now in this catalogue. The peculiar galactic X-ray source, $4 \mathrm{U} 1954+319$, has been listed in the previous editions as an HMXB. Masetti et al. (2006), however, found that the suspected field M-type giant star is indeed the counterpart of the X-ray source, based on the Chandra Observation. They suggest that $4 \mathrm{U} 1954+319$ is a wide-orbit LMXB, most likely a neutron star, accreting from the wind of an M-type giant.

1A 1246-588 was previously included in HMXB catalogue. However, RXTE observations revealed an unusual burst in the source (Levine et al. 2006). Bassa et al. (2006) recently reported an optical identification of $1 \mathrm{~A} 1246-588$ which suggests that the system is an ultracompact X-ray binary (thus a LMXB) and they note that type I bursts have been seen from this system in BeppoSAX and RXTE data.

Acknowledgements. We wish to thank Marc Ribó, Ada Paizis, Fraser Lewis, Lara Sidoli and an anonymous referee for their carefully reading the manuscript and useful comments. We also thank Marc Ribó for providing us with information on radio loud X-ray sources and Ada Paizis for access to her results prior to publication. This research has made use of the SIMBAD data base, operated at CDS, Strasbourg, France, and NASA's Astrophysics Data System (ADS). Q.Z.L. is partially supported by the Royal Science Foundation of The Netherlands, the National Natural Science Foundation of China under Grants 10673032 and 10433030.

\section{References}

Angelini, L., Loewenstein, M., \& Mushotzky, R. 2001, ApJ, 557, L35 Barnard, R., Kolb, U., \& Osborne, J. P. 2003, A\&A, 411, 553

Bassa, C. G., Jonker, P. G., in't Zand, J. J. M., \& Verbunt, F. 2006, A\&A, 446, L17

Bird, A. J., Malizia, A., Bazzano, A., et al. 2007, ApJ [arXiv: astro-ph/0611493]

Cumming, A., \& Bildsten, L. 2001, ApJ, 559, L127

Gilfanov, M. 2004, MNRAS, 349, 146

Heinke, C. O., Grindlay, J. E., Lugger, P. M., et al. 2003, ApJ, 598, 501

Jeltema, T. E., Canizares, C. R., Buote, D. A., \& Garmire, G. P. 2003, ApJ, 585, 756

Levine, A. M., Remillard, R. A., \& Galloway, D. 2006, ATel 830

Lewin, W. H. G., \& van der Klis, M. (eds) 2006, Compact Stellar X-Ray Sources (Cambridge University Press)

Liu, Q. Z., \& Mirabel, I. F. 2005, A\&A, 429, 1125 
Liu, Q. Z., van Paradijs, J., \& van den Heuvel, E. P. J. 2001, A\&A, 368, 1021 (3rd edition)

Liu, Q. Z., van Paradijs, J., \& van den Heuvel, E. P. J. 2006, A\&A, 455, 1165

Marshall, F. E., Strohmayer, T., \& Remillard, R. 1998, IAU Circ., 6891

Marti, J., Mirabel, I. F., Chaty, S., \& Rodriguez, L. F. 1998, A\&A, 330, 72

Masetti, N., Orlandini, M., Palazzi, E., et al. 2006, A\&A, 453, 295

Paredes, J. M., Marti, J., Scott, W. K., \& Zamanov, R. K. 2004, A\&A, 421, 229

Podsiadlowski, P., \& Rappaport, S. 2000, ApJ, 529, 946

Predehl, P., \& Schmitt, J. H. M. M. 1995, A\&A, 293, 889
Schatz, H., Bildsten, L., \& Cumming, A. 2003, ApJ, 583, L87

Strohmayer, T. E., \& Brown, E. F. 2002, ApJ, 566, 1045

Tauris, T. M., van den Heuvel, E. P. J., \& Savonije, G. J. 2000, ApJ, 530, L93

Verbunt, F. 2001, A\&A, 368, 137

Verbunt, F., Elson, R., \& van Paradijs, J. 1984, MNRAS, 210, 899

Verbunt, F., \& van den Heuvel, E. P. J. 1995, in X-ray Binaries, CUP, ed. W. H. G. Lewin, et al.

Winkler, C., Courvoisier, T. J.-L., Di Cocco, G., et al. 2003, A\&A, 411, L1

Woosley, S. E., \& Taam, R. E. 1976, Nature, 263, 101 